\title{
Multidrug Resistant Pseudomonas aeruginosa and Klebsiella spp Dual Isolate, causing Hospital Acquired Burn Wound Infection in Burn Care Unit of a Tertiary Care Hospital in North India
}

\author{
Iqra Majid, Nahid Nehvi and Saqib Rishi* \\ Department of Microbiology, Government Medical College Srinagar, Kashmir, India \\ *Corresponding author
}

\section{Keywords}

Hospital Acquired Infection, Burn

Wound Infection,

Pseudomonas

Aeruginosa,

Klebsiella, MDR,

Burn Care Unit

Article Info

Accepted:

04 November 2020

Available Online:

10 December 2020

\section{A B S T R A C T}

Hospital acquired infections (HAI) affect 1 in 10 patients admitted to hospital. They are associated with prolonged hospital stay, mortality and health care costs. If patients survive the initial burn and resuscitative phase, infections are a leading cause of mortality. Also Burn patients are at a higher risk of acquiring HAI with Multidrug resistant (MDR) organisms. Furthermore, infections with MDR pathogens increase morbidity, decrease treatment success, reduce hospital turn-over rate and increase cost of patient care. Samples falling under the inclusion criteria of the study and manifesting any symptoms and signs of hospital acquired burn wound infection during the management of burns were included in the study. HAI were defined based on CDC criteria. Swabs were collected from the wound showing signs of infection and were transported to microbiology laboratory for processing. Positive Isolates were confirmed by conventional biochemical tests. And isolates exhibiting ambiguous taxonomic classification were confirmed by Vitek-2 Compact Automated Identification System. Antimicrobial susceptibility testing was performed using the Kirby-Bauer disk diffusion method according to Clinical and Laboratory Standards Institute (CLSI) guidelines. A total of 71 percent (71/100) of patients developed HAI out of these 71 patients majority (74\%) developed burn wound infection .Among the total of 92 pus samples processed $80.43 \%$ were positive while as $19.56 \%$ were negative. Out of the total culture positive samples $42.3 \%$ showed multiple isolate which mostly comprised of two organisms. While as $19.56 \%$ showed no growth. Dual isolates of P.aeruginosa + Klebsiella spp constituted a majority (50\%) of the positives. P.aeruginosa alone was isolated from $16.21 \%$ samples followed by Klebsiella $(12.16 \%)$ and $E$.coli (5.4\%).All the isolates of most frequently isolated organism Pseudomonas aeruginosa were sensitive to polymyxin $-\mathrm{b} .6 .9 \%$ were sensitive to imepenem and only $4 \%$ were sensitive to levofloxicin. This isolated Pseudomonas was seen to be resistant to most of the other tested drugs. Out of the 46 Klebsiella isolates all were sensitive to polymyxin -b and to tigecycline, $15(32.6 \%)$ were sensitive to minocycine and $2(4.3 \%)$ were sensitive to ceftriaxone. HAI with MDR organisms and lack of newer antimicrobial agents with activity against them continues to be a major problem as well as a challenge for microbiologists and clinicians alike. Antibiotic stewardship, tailored infection control policies, regular screening of ICU'S are essential to combat this challenge. 


\section{Introduction}

Hospital acquired infections(HAI) are defined as infections that are not present or incubating at the time of hospital admission and develop after 48 hours or more of admission, 3 days of discharge or 30 days of an operation (ChinHong and Guglielmo, 2017; Mayhall, 2003). They affect 1 in 10 patients admitted to hospital. They are associated with prolonged hospital stay, mortality and health care costs. If patients survive the initial burn and resuscitative phase, infections are a leading cause of mortality (75\% of cases) in these patients (Lari and Alaghehbandan, 2000). The longer the patient stays in the hospital, the higher the chances for the patient to acquire hospital acquired infections in the wound (Anon Bhat and Vasaikar 2010; Church et al., 2006; Ugburo et al., 2004).

The most common types hospital acquired infection in the hospital setting are blood stream infection, urinary tract infections, pneumonias, and skin and soft tissue infections (Mayhall, 2003). Burn wound infections are one of the most important and potentially serious complications that occur in the acute period following injury. Burn wound infection is a serious problem because it causes a delay in epidermal maturation and leads to additional scar tissue formation. Invasion of microorganisms into the tissue layers below the dermis may also result in bacteremia, sepsis, and multiple-organ dysfunction syndrome (Church et al., 2006). Although the initial burn wound is sterile, immediately following thermal injury, these wounds become colonized with microorganisms (Cen et al., 2015). Gram positive bacteria that survive the thermal insult such as staphylococci located deep within sweat glands and hair follicles heavily colonize the wound surface within the first 48 hours (Soares de Macedo and Santos, 2006; Wang et al., 2010). Microorganisms transferred to a patient's skin surface via contact with contaminated external environmental surfaces, water, fomites, air, and the soiled hands of health care workers can further contaminate the wound (Cen et al., 2015). Wound colonization by yeasts and fungi usually occurs later due to the use of broad-spectrum antibiotic therapy (Soares de Macedo and Santos 2006; Wang et al., 2010) Immune suppression, intestinal bacterial translocation, extended hospitalization and invasive diagnostic and therapeutic procedures including intubation and catheterization can all contribute to contamination of burn wounds and development of systemic infection (Cen et al., 2015; Soares de Macedo and Santos, 2006). Changes in resistance pattern may be attributed to factors such as, cross infection, inappropriate use of antibiotics, selective pressure and even mutations in bacterial genome (Anon Lunawat et al., 2015) Prolonged courses of antibiotics, often in combination result in selection of multidrug resistant nosocomial strains (Leseva et al., 2013). Compared to other patients, burn patients are at a higher risk of acquiring hospital acquired infection from multi-drug resistant gram negative bacilli, thereby limiting the choice of empirical therapy (Anon Gang et al., 1999; Glik et al., 2012; Yali et al., 2014). In addition, the profile of organisms causing burn wound infections changes with time and geographical location, and from primarily gram positive to gram negative (Agnihotri, Gupta, and Joshi 2004; Altoparlak et al., 2004). Various factors have been shown to increase the prevalence of BWIs leading to Blood Stream Infections (BSIs) in patients and these can be grouped into two; patient and microbial factors. Patient factors include: size of the injury Total Burn Surface Area (\%TBSA), degree of burn, anatomical location, duration of hospital stay, systemic prophylaxis, co-morbidities (i.e. obesity, diabetes, immunosuppression, 
malnutrition, HIV) and the extremes of age (Al Laham et al., 2013; Ngugi, 2013). The microbial factors are: virulence, numbers of organisms, motility, extra-cellular products such as proteinases, collagenases, hyaluronidase, exotoxins and antimicrobial resistance (Edwards-Jones, Greenwood, and Manchester Burns Research Group, 2003).

Antibiotic-resistant organisms have been implicated in infections of the burn wound, blood and other anatomic sites in patients with major thermal injury (Church et al., 2006; Embil et al., 2001; Hsueh et al., 1998).

Furthermore, infections with multi-drug resistant pathogens whether in hospitals or in the community increase morbidity, decrease treatment success, reduce hospital turn-over rate and increase cost of patient care (Alebachew et al., 2012).

\section{Materials and Methods}

This prospective study was conducted in the Department of Microbiology, Government Medical College Srinagar Kashmir India. The patients admitted to burn care unit with the following criteria were included in the study: No infection at the time of admission and up to $48 \mathrm{hrs}$ (cultures negative); Length of stay in the hospital more than $48 \mathrm{hrs}$; Signs and symptoms suggestive of infection. Exclusion criteria: Patients referred from other hospitals; Infection acquired before 48 hours of admission.

Samples from patients falling under the inclusion criteria of the study and manifesting any symptoms and signs of hospital acquired infection during the management of burns were included in the study. Hospital acquired infections were defined based on CDC criteria as described below (Latham, 1996).

Surface swabs were collected from burn wounds after removing any dressings and topical antimicrobial agents and cleansing of the wound surface with sterile normal saline (Church et al., 2006). Swabs were collected from the depth of the wound showing signs of infection by Gently rolling swab over the surface of the wound approximately five times, focusing on area where there is evidence of pus or inflamed tissue. Specimen were places in the Stuarts transport media whenever indicated and transported to the microbiology laboratory for processing as soon as possible (Belba et al., 2013).

Positive Isolates were confirmed by conventional biochemical tests (Cheesbrough, 2006) and isolates exhibiting ambiguous taxonomic classification were confirmed by Vitek-2 Compact Automated Identification System following the manufacturer's instructions.

Antimicrobial susceptibility testing were performed using the Kirby-Bauer disk diffusion method according to Clinical and Laboratory Standards Institute (CLSI) guidelines (clinical laboratory standards institute, 2015) Briefly, from a pure culture, 3- 5 selected colonies of bacteria were picked and transferred to a tube containing $5 \mathrm{ml}$ sterile normal saline and mixed gently until a homogenous suspension was formed. Turbidity of the culture suspension was equilibrated to match 0.5 McFarland standards (Clinical laboratory standards institute, 2015). A sterile cotton swab was used and the excess suspension removed by gentle pressing and rotation of the swab against the inside wall surface of the tube. The swab was then used to distribute the bacteria evenly over the entire surface of Mueller-Hinton agar (MHA). The plates were incubated at $37{ }^{\circ} \mathrm{C}$ for $18-24$ hours and the diameters of the zone of inhibition around the disk were measured using verniercalipers in millimeters and interpreted according to CLS1 2015 criteria; sensitive, intermediate, and resistant. P. aeruginosa American Type 
Culture Collection (ATCC) 35218, S. aureus ATCC 25923 and E. coli ATCC 25922 were used as control organism.

Multidrug resistant (MDR) was defined as resistance to at least one antibiotic agent in three or more antimicrobial classes (Magiorakos et al., 2012).

Antimicrobial classes tested were Penicillin class (ampicllin, penicillin); Cephalosporin class (ceftazidime, ceftriaxone); Aminoglycosides class (genta-micin); Tetracycline class (Tetracycline); fluorquinolones class (ciprofloxacin); folate pathway inhibitors class (sulfamethmoxazole -trimethoprim); phenicols class (chloramphenicol); macrolides class (erythromycin) (Magiorakos et al., 2012).

\section{Results and Discussion}

A total of 100 patients were taken and 71 percent developed HAI and 29 percent didn't develop any of the HAI. 71 percent $(71 / 100)$ of patients developed HAI out of these 71 patients majority (74\%) developed burn wound infection. Among the total of 92 pus samples processed, Majority of the samples $80.43 \%$ were positive while as $19.56 \%$ were negative. Out of the total culture positive samples $42.3 \%$ showed multiple isolate which mostly comprised of two organisms (chart: 1 ). While as $19.56 \%$ showed no growth.

Table.1 Sensitivity profile of gram negative organisms isolated from pus

\begin{tabular}{|c|c|c|c|c|c|}
\hline Antibiotic & $\begin{array}{c}\text { Pseudomonas } \\
(\mathrm{n}=49)\end{array}$ & $\begin{array}{l}\text { E.coli } \\
(\mathbf{n}=4)\end{array}$ & $\begin{array}{c}\text { Acinetobacter } \\
(\mathrm{n}=2)\end{array}$ & $\begin{array}{c}\begin{array}{c}\text { Klebsiella } \\
(\mathrm{n}=46)\end{array} \\
\text { (1) }\end{array}$ & $\begin{array}{c}\text { Proteus } \\
(\mathrm{n}=3)\end{array}$ \\
\hline Ciprofloxacin & $\mathbf{0}$ & 2 & $\mathbf{0}$ & $\mathbf{0}$ & 1 \\
\hline Amikacin & $\mathbf{0}$ & 2 & 1 & $\mathbf{0}$ & - \\
\hline Imepenem & 3 & 2 & 1 & $\mathbf{0}$ & 3 \\
\hline Tigecycline & - & 4 & 2 & 46 & - \\
\hline $\begin{array}{l}\text { Piperacillin } \\
\text { /tazobactum }\end{array}$ & 2 & 1 & 1 & $\mathbf{0}$ & 3 \\
\hline Ceftazidime & $\mathbf{0}$ & - & 1 & $\mathbf{0}$ & 1 \\
\hline Meropenem & 1 & - & $\mathbf{0}$ & $\mathbf{0}$ & 2 \\
\hline Polymyxin-b & 49 & 4 & 2 & 46 & $\mathbf{0}$ \\
\hline Ceftriaxone & $\mathbf{0}$ & $\mathbf{0}$ & - & 2 & 2 \\
\hline Moxifloxacin & - & - & 1 & $\mathbf{0}$ & - \\
\hline $\begin{array}{l}\text { Amoxicillin + } \\
\text { clavulinic acid }\end{array}$ & - & 1 & 2 & 1 & 1 \\
\hline Ampicillin /sulbactum & - & $\mathbf{0}$ & 1 & - & - \\
\hline Gentamicin & $\mathbf{0}$ & 3 & $\mathbf{0}$ & $\mathbf{0}$ & - \\
\hline Ertapenem & - & 3 & - & - & 2 \\
\hline Cotrimoxazole & - & 2 & - & $\mathbf{0}$ & $\mathbf{0}$ \\
\hline Tobramycin & $\mathbf{0}$ & $\mathbf{0}$ & $\mathbf{0}$ & $\mathbf{0}$ & - \\
\hline Cefepime & $\mathbf{0}$ & $\mathbf{0}$ & 1 & $\mathbf{0}$ & 1 \\
\hline Aztreonem & - & - & 1 & 1 & 1 \\
\hline Levofoxacin & 2 & 3 & $\mathbf{0}$ & 1 & - \\
\hline Minocycline & - & - & - & 15 & - \\
\hline Cefotaxime & - & $\mathbf{0}$ & 1 & - & - \\
\hline
\end{tabular}


Chart.1 Frequency distribution of aerobic isolates from pus samples

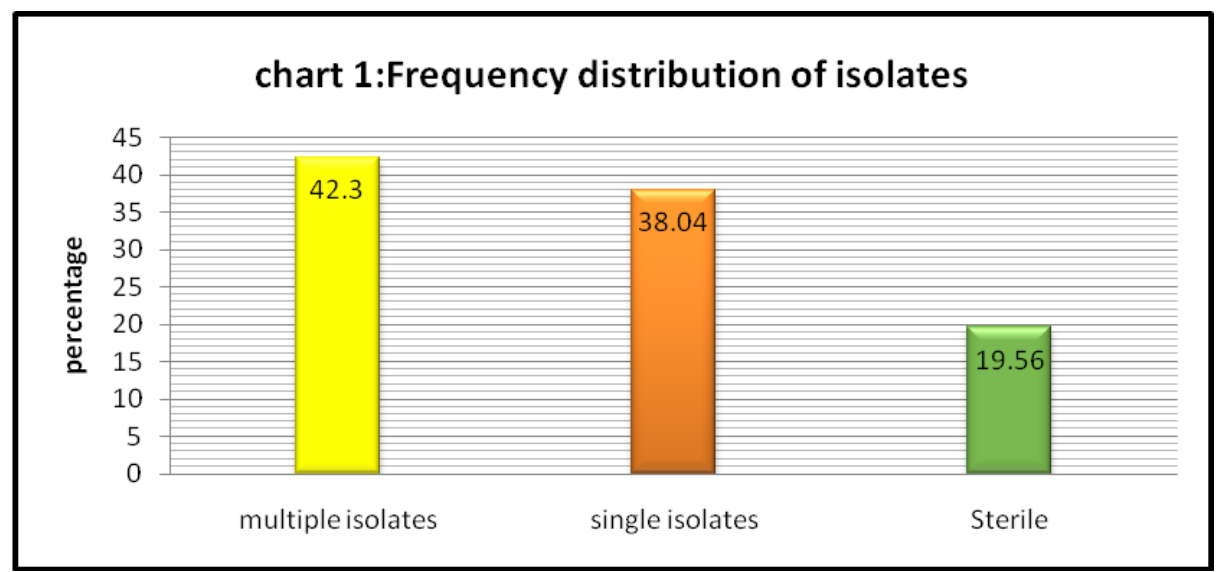

Chart.2 Frequency distribution of organisms isolated from pus swabs

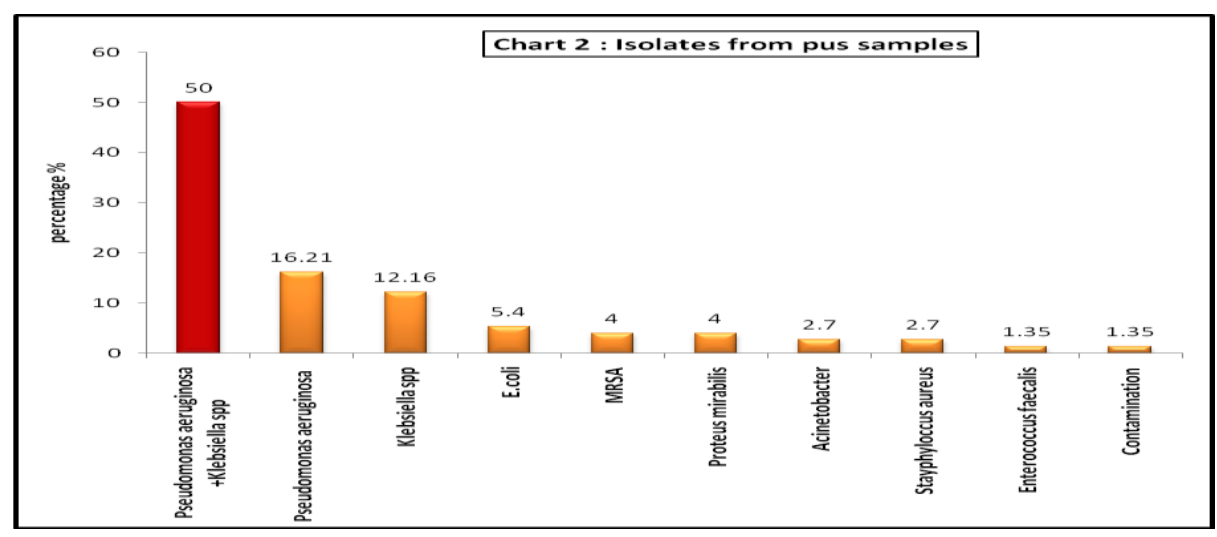

Dual isolates of P.aeruginosa + Klebsiella spp constituted a majority $(50 \%)$ of the positives. $\mathrm{P}$. aeruginosa alone was isolated from $16.21 \%$ samples followed by Klebsiella (12.16\%) and E. coli (5.4\%). (Chart: 2)

Sensitivity profile of organisms isolated from pus: All the isolates of most frequently isolated organism Pseudomonas aeruginosa were sensitive to polymyxin -b. $6.9 \%$ were sensitive to imepenem and only $4 \%$ were sensitive to levofloxicin. This isolated Pseudomonas was seen to be resistant to most of the other tested drugs. Out of the 46 Klebsiella isolates all were sensitive to polymyxin $-\mathrm{b}$ and to tigecycline, $15(32.6 \%)$ were sensitive to minocycine and $2(4.3 \%)$ were sensitive to ceftriaxone (Table 1).
HAI are a significant problem for health services in all countries, with important effects on the survival of high-risk patients, such as burn patients. Infections of burn sites are very dangerous problems that can compromise the patient's survival and the outcome of reconstructive treatment (Anon n.d.).

Among the $100 \mathrm{HAI}$ in our study, burn wound infection constituted $74 \%$,. Our study indicates here that the rate of development of HAI are substantially higher than similar studied from India (Taneja et al., 2004) and also from other parts of the world $(58,69)$.

Infection remains a foremost concern in the management of burn wounds because the 
large raw area with its serous exudate may act as a huge culture plate on which organisms can establish and multiply (Gang et al., 1999). Fresh burns are usually sterile but progressively become colonized with one or more bacterial species as the patient's stay in burn unit increases.

In our study, $38.04 \%$ of swab cultures yielded single isolates whereas multiple isolates were seen in $42.3 \%$ of the swab cultures. Overall, $P$. aeruginosa+ Klebsiella were the commonly isolated organism $(50 \%)$ followed by pseudomonas alone (16.2\%), Klebsiella alone (12.16\%), E.coli (5.4\%) MRSA (4\%) and Acinetobacter spp. (2.70\%) Pseudomonas spp has been found to be the major pathogen $(66.2 \%)$ in burn patients and was also the most important cause of sepsis in them. As far as, the overall colonizing organism is concerned our findings are consistent with those reported by Kaushik et al., (Revathi, Puri, and Jain 1998). These authors reported that Pseudomonas was the most commonly cultured organism (54.2\%) followed by $S$. aureus (20.8\%). Similar observations were made by Revathi et al., who reported $P$. aeruginosaas the most common isolate (36\%) from the burn patients followed by $S$. aureus (19\%). Our observations are incontrast to a number of studies from various countries which reported $S$. aureus as the most common colonizing organisms in their patients et al., 1992) Ozumba and Jiburum (Ozumba and Jiburum, 2000) from Africa however, reported most common colonizing organism as Klebsiella species (26.7\%) followed by S. aureus $(25.6 \%)$.

In pus samples, commonly isolated organism $P$. aeruginosa was sensitive to polymyxin B in all the isolates (100\%), 6.9\% were sensitive to imipenem and only $4 \%$ were sensitive to levofloxacin. Out of the 46 Klebsiella isolates from pusall were sensitive to polymyxin $-b$, $15(32.6 \%)$ were sensitive to minocycine and
2(4.3\%) were sensitive to ceftriaxone. Hospital acquired isolates of Klebsiella are resistant to most antibiotic as a result of acquisition of multidrug resistance plasmids.

Klebsiella is known to carry plasmids encoding extended spectrum beta lactamases and carbapenemases and treatment with such strains is associated with treatment failure and death.

This study provides assessment of important aspects of hospital acquired burn wound infections. Gram negative organisms predominated in hospital acquired burn wound infections, with $P$. aeruginosa+ Klebsiella spp dual isolate being the most common. We found these isolates showed multiple drug resistance to commonly prescribed antimicrobial agents.

This study concludes that HAI's with MDR organisms in particular continues to be a major problem for the healthcare facilities and a challenge for the microbiologists and clinicians alike. Lack of newer antimicrobial agents with activity against MDR organisms makes periodic studies on antimicrobial susceptibility patters very important. Were commend regular screening of ICU's to give early warnings for the presence of MDR pathogens. Antibiotic stewardship and tailored infection control policies for each institution are essential to combat this challenge.

\section{References}

Agnihotri, N., V. Gupta, and R. M. Joshi. 2004. Aerobic Bacterial Isolates from Burn Wound Infections and Their Antibiograms--a Five-Year Study. Burns: Journal of the International Society for Burn Injuries 30(3):241-43. Al Laham, N. A., A. A. Elmanama, and G. A. Tayh. 2013. Possible Risk Factors 
Associated with Burn Wound Colonization in Burn Units of Gaza Strip Hospitals, Palestine. Annals of Burns and Fire Disasters 26(2):68-75. Alebachew, Tigist, Gizachew Yismaw, Ayelegn Derabe, and Zufan Sisay. 2012. Staphylococcus Aureus Burn Wound Infection Among Patients Attending Yekatit 12 Hospital Burn Unit, Addis Ababa, Ethiopia. Ethiopian Journal of Health Sciences 22(3):20913.

Altoparlak, Ulku, Serpil Erol, Mufide N. Akcay, Fehmi Celebi, and Ayten Kadanali. 2004. The Time-Related Changes of Antimicrobial Resistance Patterns and Predominant Bacterial Profiles of Burn Wounds and Body Flora of Burned Patients. Burns: Journal of the International Society for Burn Injuries 30(7):660-64.

Anon. n.d. Bacterial and Fungal Colonization of Burn Wounds. - PubMed - NCBI. Retrieved September 18, 2018a (https://www.ncbi.nlm.nih.gov/pubmed /16184232).

Anon. n.d. Bacterial Infections in Burn Patients at a Burn Hospital in Iran. PubMed - NCBI. Retrieved October 27 , $2018 b$ (https://www.ncbi.nlm.nih.gov/pubmed /18219081).

Anon. n.d. Burns Injury in Children: Is Antibiotic Prophylaxis Recommended? Chahed J, Ksia A, Selmi W, Hidouri S, Sahnoun L, Krichene I, Mekki M, Nouri A - Afr J Paediatr Surg. Retrieved September 13, 2018c (http://www.afrjpaedsurg.org/article.as p? issn $=0189$ -

6725; year $=2014 ;$ volume $=11 ;$ issue $=4 ; \mathrm{s}$ page $=323$; epage $=325$; aulast $=$ Chahed) .

Anon. n.d. Microbial Profile of Burn Wound Infections in Burn Patients, Taif, Saudi Arabia Insight Medical Publishing. Retrieved September 13, 2018d (http://www.acmicrob.com/microbiolo gy/microbial-profile-of-burn-woundinfections-inburn-patients-taif-saudiarabia.php?aid=8879).

Anon. n.d. Relationship between Antimicrobial Resistance and Patient Outcomes: Mortality, Length of Hospital Stay, and Health Care Costs | Clinical Infectious Diseases | Oxford Academic. Retrieved October 27, $2018 \mathrm{e}$

(https://academic.oup.com/cid/article/4 2/Supplement_2/S82/377684).

Atoyebi, O. A., G. O. A. Sowemimo, and T. Odugbemi. 1992. Bacterial Flora of Burn Wounds in Lagos, Nigeria: A Prospective Study. Burns 18(6):44851.

Belba, Monika Kristaq, Elizana Ylber Petrela, and Amy Gjergji Belba. 2013. Epidemiology of Infections in a Burn Unit, Albania. Burns: Journal of the International Society for Burn Injuries 39(7):1456-67.

Bhat, V. G. and S. D. Vasaikar. 2010. Bacteriological Profile and Antibiogram of Aerobic Burn Wound Isolates in Mthatha, Eastern Cape, South Africa. Southern African Journal of Epidemiology and Infection 25(4):16-19.

Cen, Hanghui, Zhenbo Wu, Fan Wang, and Chunmao Han. 2015. Pathogen Distribution and Drug Resistance in a Burn Ward: A Three-Year Retrospective Analysis of a Single Center in China. International Journal of Clinical and Experimental Medicine 8(10):19188-99.

Cheesbrough, Monica. 2006. District Laboratory Practice in Tropical Countries. Leiden: Cambridge University Press.

Chin-Hong, Peter V. and B. Joseph Guglielmo. 2017. Common Problems in Infectious Diseases \& Antimicrobial 
Therapy. in Current Medical Diagnosis \& Treatment 2018, edited by M. A. Papadakis, S. J. McPhee, and M. W. Rabow. New York, NY: McGraw-Hill Education.

Church, Deirdre, Sameer Elsayed, Owen Reid, Brent Winston, and Robert Lindsay. 2006. Burn Wound Infections. Clinical Microbiology Reviews 19(2):403-34.

clinical laboratory standards institute. 2015.in M100-S25: Performance Standards for Antimicrobial Susceptibility Testing; Twenty-Fifth Informational Supplement. Vol. 25th information supplement. wayne, pennsylvania: cilnical and laboratory standards institite.

Edwards-Jones, Valerie, John E. Greenwood, and Manchester Burns Research Group. 2003. What's New in Burn Microbiology? James Laing Memorial Prize Essay 2000. Burns: Journal of the International Society for Burn Injuries 29(1): 15-24.

Embil, J. M., J. A. McLeod, A. M. Al-Barrak, G. M. Thompson, F. Y. Aoki, E. J. Witwicki, M. F. Stranc, A. M. Kabani, D. R. Nicoll, and L. E. Nicolle. 2001. An Outbreak of Methicillin Resistant Staphylococcus Aureus on a Burn Unit: Potential Role of Contaminated Hydrotherapy Equipment. Burns: Journal of the International Society for Burn Injuries 27(7): 681-88.

Gang, R. K., R. L. Bang, S. C. Sanyal, E. Mokaddas, and A. R. Lari. 1999. Pseudomonas aeruginosa Septicaemia in Burns. Burns: Journal of the International Society for Burn Injuries 25(7): 611-16.

Glik, Justyna, Marek Kawecki, Tadeusz Gaździk, and Mariusz Nowak. 2012. The Impact of the Types of Microorganisms Isolated from Blood and Wounds on the Results of
Treatment in Burn Patients with Sepsis. Polski Przeglad Chirurgiczny 84(1):616.

Hsueh, P. R., L. J. Teng, P. C. Yang, Y. C. Chen, S. W. Ho, and K. T. Luh. 1998. Persistence of a Multidrug-Resistant Pseudomonas Aeruginosa Clone in an Intensive Care Burn Unit. Journal of Clinical Microbiology 36(5):1347-51.

Lari, A. R. and R. Alaghehbandan. 2000. Nosocomial Infections in an Iranian Burn Care Center. Burns: Journal of the International Society for Burn Injuries 26(8):737-40.

Latham, Robert H. 1996. Hospital Epidemiology and Infection Control, CG Mayhall, Baltimore, Maryland: Williams and Wilkins, 1996; 1,283 Pages. Infection Control \& Hospital Epidemiology 17(5):1-14.

Leseva, M., M. Arguirova, D. Nashev, E. Zamfirova, and O. Hadzhyiski. 2013. Nosocomial Infections in Burn Patients: Etiology, Antimicrobial Resistance, Means to Control. Annals of Burns and Fire Disasters 26(1):511.

Lunawat, Ajay, Rajesh Sharma, Venkatesh Kolla, and Shailendra Patel. 2015. Emerging Resistance of Higher Antimicrobials and Growing Sensitivity of Old Antimicrobials against Existing Infections in Burns. International Surgery Journal 385-91.

Magiorakos, A. P., A. Srinivasan, R. B. Carey, Y. Carmeli, M. E. Falagas, C. G. Giske, S. Harbarth, J. F. Hindler, G. Kahlmeter, B. Olsson-Liljequist, D. L. Paterson, L. B. Rice, J. Stelling, M. J. Struelens, A. Vatopoulos, J. T. Weber, and D. L. Monnet. 2012. MultidrugResistant, Extensively Drug-Resistant and Pandrug-Resistant Bacteria: An International Expert Proposal for Interim Standard Definitions for Acquired Resistance. Clinical 
Microbiology and Infection: The Official Publication of the European Society of Clinical Microbiology and Infectious Diseases 18(3):268-81.

Mayhall, C. Glen. 2003. The Epidemiology of Burn Wound Infections: Then and Now. Clinical Infectious Diseases: An Official Publication of the Infectious Diseases Society of America 37(4):543-50.

Ngugi, Moses G. 2013. Correlation of Burn Wound Infection and Mortality of Burn Injury Patients Hospitalized at Kenyatta National Hospital. Thesis, University of Nairobi.

Oncul, O., E. Ulkur, A. Acar, V. Turhan, E. Yeniz, Z. Karacaer, and F. Yildiz. 2009. Prospective Analysis of Nosocomial Infections in a Burn Care Unit, Turkey. The Indian Journal of Medical Research 130(6): 758-64.

Ozumba, U. C. and B. C. Jiburum. 2000. Bacteriology of Burn Wounds in Enugu, Nigeria. Burns: Journal of the International Society for Burn Injuries 26(2): 178-80.

Revathi, G., J. Puri, and B. K. Jain. 1998. Bacteriology of Burns. Burns: Journal of the International Society for Burn Injuries 24(4): 347-49.

Soares de Macedo, Jefferson Lessa and João

Barberino Santos. 2006. Nosocomial Infections in a Brazilian Burn Unit.
Burns: Journal of the International Society for Burn Injuries 32(4):477-81. Taneja, Neelam, Rekha Emmanuel, P. S. Chari and Meera Sharma. 2004. A Prospective Study of HospitalAcquired Infections in Burn Patients at a Tertiary Care Referral Centre in North India. Burns: Journal of the International Society for Burn Injuries 30(7):665-69.

Ugburo, A. O., O. A. Atoyebi, J. O. Oyeneyin, and G. O. A. Sowemimo. 2004. An Evaluation of the Role of Systemic Antibiotic Prophylaxis in the Control of Burn Wound Infection at the Lagos University Teaching Hospital. Burns: Journal of the International Society for Burn Injuries 30(1): 43-48.

Wang, Yu, Hong-Tai Tang, Zhao-Fan Xia, Shi-Hui Zhu, Bing Ma, Wei Wei, Yu Sun, and Kai-Yang Lv. 2010. Factors Affecting Survival in Adult Patients with Massive Burns. Burns: Journal of the International Society for Burn Injuries 36(1):57-64.

Yali, Gong, Chen Jing, Liu Chunjiang, Zhang Cheng, Luo Xiaoqiang, and Peng Yizhi. 2014. Comparison of Pathogens and Antibiotic Resistance of Burn Patients in the Burn ICU or in the Common Burn Ward. Burns: Journal of the International Society for Burn Injuries 40(3): 402-7.

\section{How to cite this article:}

Iqra Majid, Nahid Nehvi and Saqib Rishi. 2020. Multidrug Resistant Pseudomonas aeruginosa and Klebsiella spp Dual Isolate, causing Hospital Acquired Burn Wound Infection in Burn Care Unit of a Tertiary Care Hospital in North India. Int.J.Curr.Microbiol.App.Sci. 9(12): 210218. doi: https://doi.org/10.20546/ijcmas.2020.912.028 